\title{
Competitividad en el turismo cultural: estudio de caso *
}

\author{
Competitiveness in Cultural Tourism: Case Study \\ Yuselim Angélica Villa-Hernández (iD \\ Maestra en Administración, Universidad Michoacana de San Nicolás de Hidalgo, \\ Morelia-México,yuselim.23@gmail.com \\ Carlos Merino-Moreno \\ Doctor en Contabilidad y Organización, Universidad Autónoma de Madrid, \\ Madrid-España, carlos.merino@uam.es \\ Virginia Hernández-Silva \\ Doctora en Ciencias, Universidad Michoacana de San Nicolás de Hidalgo, \\ Morelia-México,vhsilva_17@hotmail.com
}

\begin{abstract}
Cómo citar / How to cite
Villa-Hernández, Y. A.; Merino-Moreno, C.; Hernández-Silva, V. (2021). Competitividad en el turismo cultural: estudio de caso. Revista CEA, v. 7, n. 14, e1619. https://doi.org/10.22430/24223182.1619
\end{abstract}

Recibido: 11 de diciembre de 2020

Aceptado: 29 de marzo de 2021

\section{Resumen}

Las empresas son sistemas compuestos por recursos y capacidades; es decir, sistemas con activos tangibles e intangibles. Éstos últimos, los intangibles, son el objeto de esta investigación, la cual se enmarca en una perspectiva exploratoria y cualitativa, la cual busca identificar los activos intangibles asociados a tres factores clave de las organizaciones y de las empresas con orientación al sector turístico cultural: la organización, la mercadotecnia y la satisfacción del cliente como sujeto particular; lo anterior, teniendo como punto de referencia para este estudio, la ciudad de Morelia, en México. La investigación pretende generar conocimiento para dichas empresas y contribuir en los procesos administrativos que aporten competitividad al sector turístico en México. Entre los principales resultados que arrojó el estudio, se encuentra que estas empresas pueden aprovechar mejor los activos intangibles que tienen a su disposición en los tres factores anteriormente mencionados. Como conclusión final, se plantea la importancia que tienen esos activos intangibles en las empresas del sector turístico en México para su mejor desempeño y, de igual forma, se identifica la oportunidad para continuar este estudio de manera empírica con una propuesta de

\footnotetext{
* Este artículo se deriva del proyecto titulado «Competitividad en el turismo cultural: estudio de caso» y ha sido financiado con recursos del Consejo Nacional de Ciencia y Tecnología - Conacyt.
} 
modelo de competitividad turística que permita enriquecer el estado del arte de esta línea de investigación en México.

Palabras clave: competitividad, turismo cultural, capital intelectual, mercadotecnia, satisfacción del turista.

Clasificación JEL: M10, L83, D24.

\title{
Highlights
}

- Los activos intangibles contribuyen a la construcción de la competitividad y pueden garantizar el éxito en los mercados turísticos.

- El activo intangible de la organización se encuentra en el capital humano, mientras que en la mercadotecnia se ve reflejado en el capital relacional.

- Los prestadores de servicios turísticos deben poner mayor énfasis en la atención al turista para lograr la satisfacción del mismo y conseguir su fidelidad.

\begin{abstract}
Companies are systems composed of resources and capabilities, that is, they have tangible and intangible assets. The objective of this exploratory and qualitative study is to identify the intangible assets associated with three key factors of organizations and companies, i.e., organization, marketing, and customer satisfaction, in the cultural tourism sector in Morelia, Mexico. This study aims to generate knowledge for such companies and contribute to administrative processes that improve the competitiveness of the Mexican tourism sector. It was found that this type of companies can better exploit the intangible assets they already have in relation to the three factors mentioned above. The final conclusion is that intangible assets are important for companies in the Mexican tourism sector in order to improve their performance. This study can be complemented with an empirical proposal of a tourism competitiveness model that can advance this field of research in Mexico.
\end{abstract}

Keywords: Competitiveness, cultural tourism, intellectual capital, marketing, tourist satisfaction.

JEL classification: M10, L83, D24.

\section{Highlights}

- Intangible assets contribute to the construction of competitiveness and can ensure success in tourism markets.

- The intangible assets of organizations are found in their human capital; in marketing, such asset is reflected in the relational capital.

- Tourism service providers should further emphasize tourist assistance to achieve customer satisfaction and loyalty. 


\section{INTRODUCCIÓN}

Según el Foro Económico Mundial (WEF, por sus siglas en inglés de World Economic Forum), México ocupa el decimonoveno lugar en el ranking de competitividad de viajes y turismo (WEF, 2019). Lo anterior supone la necesidad de buscar estrategias y modelos que puedan ayudar al sector turístico (con fuerte componente cultural) a mejorar dicho índice y posicionar al país en un lugar más alto en el escalafón mencionado. Así, este trabajo es un estudio relativo al turismo cultural, dada su importancia en el mundo, y particularmente en la ciudad de Morelia, México. Además, las actividades turísticas aportan buena parte de los empleos y riqueza a la economía de esta región y de la nación (Corte-Cruz, 2019).

El sector del turismo en el país aporta $8.7 \%$ de riqueza al Producto Interno Bruto (PIB) nacional, y la ciudad de Morelia está conformada económicamente por micro, pequeñas y medianas empresas (MiPymes) del sector turismo. De acuerdo a los datos del Instituto Nacional de Estadística y Geografía, (INEGI), la esperanza de vida de los negocios en México es de 7.8 años en promedio (INEGI, 2016). Por lo tanto, es imprescindible para la ciudad de Morelia incrementar los niveles de competitividad de las MiPymes que soportan el sector del turismo.

El artículo está conformado de la siguiente manera: en la primera parte del trabajo, como introducción, se plantea la importancia del turismo en México. La segunda parte se compone por el marco teórico, integrado por conceptos encontrados en la literatura sobre la competitividad turística y los activos intangibles. El tercer apartado describe la metodología empleada en la investigación. Por último, el cuarto punto muestra como resultado de la investigación documental una propuesta de modelo de competitividad turística. Además, se expone la discusión y conclusiones del trabajo de investigación.

\section{MARCO TEÓRICO}

\section{Competitividad turística en México}

EI WEF (Uppink Calderwood \& Soshkin, 2019) realiza de forma bienal, desde el año 2006, el índice de competitividad de viajes y turismo. La finalidad es medir los factores y políticas que hacen posible el desarrollo sostenible del sector turístico. Dicho desarrollo permite medir la competitividad de 140 países en cuanto a su capacidad turística. Para el cálculo se utilizan 14 pilares que tienen 90 indicadores individuales. En el más reciente reporte de competitividad turística publicada por el Foro Económico Mundial (WEF, 2019), México se encuentra en el lugar decimonoveno, subiendo tres lugares en el índice con respecto al año 2017, y once lugares más que en el 2015. México obtiene una puntuación de 4.7, siendo España el primer lugar (por tercera vez consecutiva) con una puntuación de 5.4 .

Cabe destacar que México ocupa el primer lugar del índice de 140 países en cuanto a sus recursos naturales. Obtiene el décimo lugar del índice por sus recursos culturales y turismo de negocios, y ocupa el lugar 29 en cuanto a la priorización del turismo. Los tres indicadores antes mencionados son los que mayor calificación le aportan a la competitividad turística de México. Los indicadores con menor calificación obtenida son la seguridad, donde ocupa el lugar 126 del índice; la sustentabilidad 
ambiental, con el lugar 108; el ambiente empresarial ocupa el lugar 98; y el lugar 81 en preparación de las TICs para el turismo (WEF, 2019).

\section{Importancia del turismo en México}

De acuerdo con WEF, para el año 2018 los ingresos recibidos por el turismo internacional en México fueron US 21336000 000, aportando el 8.0\% al PIB. Ese mismo año, el sector turístico ocupó al 8.6\% de la población, generando un total de 4568400 empleos (WEF, 2019). En años recientes, el turismo tendía hacia el alza, manteniéndose como una industria en crecimiento que impulsaba el resto de la actividad económica, así lo reconoce la Secretaría de Turismo en México (Coordinación de Asesores del Secretario de Turismo, 2018).

La cantidad de ramas económicas que el turismo impacta es grande. Los visitantes requieren de transporte, alimento, hospedaje, servicios de comunicación, esparcimiento, servicios médicos, entre otros (Coordinación de Asesores del Secretario de Turismo, 2018; Djukic \& Vukmirovic, 2012). El turismo en México aporta empleos para más de 4 millones de personas de forma directa. El beneficio es mayor: la Organización Mundial del Turismo (OMT) estima que por cada empleo directo en el turismo se generan alrededor de 1.5 empleos adicionales de manera indirecta. Bajo este supuesto, en México otros 6 millones de personas son empleadas de manera indirecta por el turismo, para llegar a 10 millones de fuentes de ingresos (Coordinación de Asesores del Secretario de Turismo, 2018).

Pero, la pandemia de COVID-19 llegó al mundo para cambiar todas las anteriores tendencias de crecimiento en el sector turismo. Al implementar estrategias de salud públicas basadas en el confinamiento de las personas y la baja movilidad de la población, llevó al cierre de las empresas del sector turístico. Según el último Barómetro del Turismo Mundial, las llegadas internacionales cayeron un $81 \%$ en julio y un $79 \%$ en agosto, precisamente los meses que suelen ser los de mayor movimiento del año y que coinciden con la época de verano (Organización Mundial del Turismo, 2020).

En México se mantuvieron cerrados los hoteles por un periodo de cuatro meses, fue hasta julio del 2020 que las autoridades competentes permitieron la reapertura de los mismos. Para el mes de agosto se reportó que la ocupación hotelera fue del 25\% (Unidad de Asuntos y Cooperación Internacionales, 2020). El Consejo Nacional Empresarial Turístico (CNET) prevé una caída del $48 \%$ del PIB del sector este año, mientras que la Secretaría de Turismo estima que no se recuperará por completo hasta 2023 (Migueles, 2020). Las estimaciones de reducción del PIB nacional están alrededor del $7.0 \%$ para el año 2020 y la caída en el PIB turístico se estima en 3.7\%, lo que significaría que la mayor parte de la contracción de la economía se debiera por el derrumbe del sector turístico (CNET \& CICCTUR, 2020). En opinión de Miguel Torruco, titular de la Secretaría de Turismo (Sectur), la reactivación del turismo se basará en la seguridad sanitaria, marketing y promoción, así como en una mayor resiliencia en el sector (El Universal, 2020).

\section{Competitividad del destino turístico}

Como parte fundamental del aporte de este estudio, el factor de competitividad del destino turístico se convierte en el marco de referencia al que se unirán las variables asociadas a los activos intangibles relativas a la organización, la mercadotecnia y la satisfacción del cliente (Teixeira et al., 2019). 
Abordando la competitividad en el ámbito turístico, Hassan (2000) la define como «la capacidad de un destino para crear e integrar productos con valor añadido que permitan sostener los recursos locales y conservar su posición de mercado respecto a sus competidores» (p. 113).

Asimismo, Crouch y Brent Ritchie (1999) definen a la competitividad turística como «la capacidad de un país para crear valor añadido e incrementar, de esta forma, el bienestar nacional mediante la gestión de ventajas y procesos, atractivos, agresividad y proximidad, integrando las relaciones entre los mismos en un modelo económico y social» (p. 144). Del concepto anterior, se identifica que son diversos los factores que influyen para hacer a un destino más competitivo, modelo que Ibáñez Pérez (2011) aprovecha para determinar dichos factores para la competitividad del Sector Turístico en México (ver Figura 1).

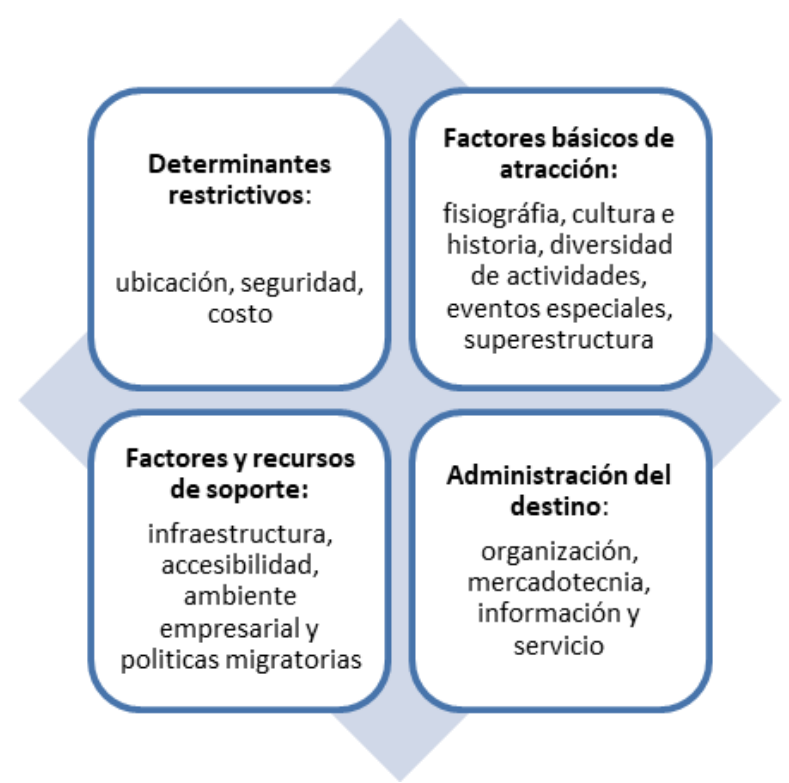

Figura 1. Modelo de competitividad turística de Crouch y Ritchie

Figure 1. Crouch and Ritchie's tourism competitiveness model Fuente: elaboración propia a partir de (Crouch \& Brent Ritchie, 1999; Ibáñez Pérez, 2011).

Los factores determinantes restrictivos son aquellos que limitan la demanda del turista, es decir, que influyen de manera negativa al momento del turista tomar una decisión cuando de escoger un destino turístico se trata. Por otro lado, los factores básicos de atracción como el clima, el paisaje, la flora y la fauna se relacionarían con la capacidad del destino para utilizar estos recursos a largo plazo. Desarrollar su competitividad a lo largo del tiempo depende de la calidad de la gestión, de trabajadores, niveles de servicio y política gubernamental (Bobirca \& Cristureanu, 2015). Los factores de soporte se componen por la infraestructura que exista en el lugar del destino, tal como vías aéreas o de transporte que faciliten la llegada del turista a su destino. Por último, los factores de administración del destino están integrados por la organización, mercadotecnia e información y servicio de las empresas de servicios turísticos del destino (Crouch \& Brent Ritchie, 1999).

La herencia cultural de un destino es crucial para la prosperidad a largo plazo y ayuda a fortalecer el sentido de pertenencia y el orgullo cívico de los residentes (Chu \& Uebegang, 2002; Gómez Vega \& Herrero Prieto, 2017). Cuanto más orgullosos están los residentes de su ciudad natal, más dispuestos 
están a compartir sus hogares con los turistas. Los turistas desean experimentar culturas nuevas o diferentes (Báez-Montenegro \& Devesa-Fernández, 2017). Al viajar, los turistas tienen la oportunidad de ponerse en el lugar de otras personas. Esto les permite olvidar sus problemas, bajar los niveles de estrés y ver las cosas como lo harían los lugareños. Más allá de los aspectos económicos y políticos, como argumentan Brent Ritchie \& Crouch (2003), un destino turístico es un lugar que las personas prefieren visitar debido a su experiencia única que no se encuentra en ningún otro lugar. Este elemento es su ventaja competitiva.

Siguiendo el modelo de Crouch y Brent Ritchie (1999), para lograr la competitividad, las empresas del sector turístico deben poner énfasis en sus estrategias de organización, mercadotecnia e información y servicio (satisfacción del cliente), de ahí que la presente investigación centre su objeto de estudio en este factor de administración de la empresa de servicios turísticos, considerando el análisis de la competitividad derivada a través de su relación con los activos intangibles de la empresa turística. Ahora más que nunca, los gobiernos y las partes interesadas de la industria del turismo deben considerar los costos, riesgos e impactos de los peligros ambientales globales en los viajes y el turismo.

\section{Los activos intangibles}

Desde la consideración de la Teoría de Recursos y Capacidades, de Hamel y Prahalad (1990), se ha venido configurando una línea de investigación clara en el ámbito de los activos intangibles como pilares donde se concentra la aportación de valor en las organizaciones, dado que los recursos solo generan rendimiento ante una buena disposición de intangibles basados en actitudes, conocimientos, experiencia, etcétera. Asimismo, Barber y Darder (2004) sostiene que los recursos financieros, tecnológicos y materiales por sí solos no pueden generar ventajas competitivas, por lo que el recurso humano es imprescindible para las organizaciones. En el personal de una empresa residen los conocimientos, y es el personal quien tiene la capacidad de poder gestionar los recursos para la organización (Fitz Patrick et al., 2013).

En línea con esta teoría, se evidencia que los activos intangibles son los conductores de ventaja competitiva, dado que las empresas rivales encuentran más difícil imitar activos intangibles que los activos físicos o tangibles (Ochoa Hernández et al., 2012; García-Gallo et al., 2020). Es por ello que la teoría de recursos y capacidades deja ver el valor de los recursos intangibles. Desde un marco conceptual, Bueno Campos (1998) define a los activos intangibles como «todo aquello de lo que se sirve y utiliza una empresa para crear valor, pero que no es posible contabilizar dada su naturaleza no tangible» (p.114). Desde la aparición de esta teoría, Grant (1991) afirmaba que ese resurgimiento por el interés de analizar los recursos de la empresa e incluirlos en la formulación de sus estrategias, es derivado de la insatisfacción ocasionada por el marco de equilibrio estático proporcionado por la organización industrial, donde los esquemas de dirección y gestión se centran en una economía de tangibles.

\section{Los activos intangibles en la organización}

De acuerdo con Miles y Quintillán (2005), los activos intangibles generan valor al momento de combinarse. Cada organización posee una serie de intangibles que están vinculados y articulados de una forma particular. Por ello, dos empresas con los mismos intangibles pueden tener resultados 
diferentes debido a la forma como los articulan (Zaragoza-Sáez et al., 2020). La ventaja competitiva que puede lograr la unión de los intangibles que posee una empresa es mayor a la que se puede obtener de manera individual. Por tanto, los resultados de la organización están determinados, en gran parte, por el sistema de sus activos intangibles (Bontis, 2001).

Chen et al., (2004) sostienen que, bajo las circunstancias competitivas de la actualidad, una empresa debe luchar por una posición dominante para la supervivencia y el desarrollo en la competencia de aprendizaje entre las empresas. La gestión de capital intelectual se ha convertido en el núcleo de la gestión empresarial en la era de la economía del conocimiento. Para Hernández (2011), el capital intelectual permite a las empresas obtener utilidades por las ideas e innovaciones de sus empleados, siempre y cuando los puedan proteger y los puedan administrar; es decir, obtener ventajas competitivas irrepetibles, ventajas imposibles de imitar.

El conocimiento que posee una organización debe potenciarse con el uso del conocimiento disponible externamente, lo que supone abrir una serie de conexiones con fuentes y grupos de interés, creando un marco relacional estratégico para el conocimiento. Así surge la composición internacionalmente reconocida para el capital intelectual, que consta de capital humano, capital estructural y capital relacional (Bueno et al., 2008; Bueno et al., 2011). A medida que las empresas se preocupan por analizar su estructura organizacional, se producen mejoras y desarrollo de nuevos procesos de creación de valor. De igual manera, una medición de los componentes del capital intelectual permite identificar en qué nivel están frente a la competencia en cuanto a elementos como lo humano, lo estructural y lo relacional (Gómez-Bayona et al., 2020).

En este sentido, el factor organizacional asociado a los intangibles está reflejado en el capital humano y en el capital estructural. El capital humano es un componente del capital intelectual que requiere un análisis de factores como los elementos motivacionales (sentido de pertenencia, compromiso, satisfacción, espíritu creativo y emprendedor), además de elementos aptitudinales (formación, especialización y actualización de competencias), e incluso elementos asociados a la experiencia (trayectoria, dominio y visión internacional). Por el lado del capital estructural, se consideran variables como el diseño organizacional, la existencia de procesos organizacionales, la utilización de las TICs y la configuración del esfuerzo innovador (Bueno et al., 2011; Rodríguez Antón \& Rubio Andrada, 2006).

\section{Los activos intangibles en la mercadotecnia}

La mercadotecnia, o marketing, es una disciplina que tiene un fundamento histórico cuyo origen no se genera de manera casual, corresponde más bien a un proceso de evolución acaecido dentro del desarrollo económico de la humanidad (Hoyos, 2008). Una actividad empresarial con éxito no sería posible sin el uso de herramientas de marketing (Larina \& Romanenko, 2015). El desarrollo mismo del mercado, y por ende del capitalismo, ha llevado a que se demanden estrategias de marketing más particulares. El marketing es un vector en el que convergen los actores económicos y sociales cuyos motivos han generado cambios sociales, los cuales, a su vez, exigen una revalorización constante del campo de estudio y del objeto de estudio (Shaw \& Brian Jones, 2005).

En este ámbito de análisis es imprescindible incorporar la variable Internet, dado que ha modificado la dinámica del mercado y, por ende, del consumo. La naturaleza de la Internet y sus efectos, tanto 
en el comportamiento del consumidor, como en los modelos de negocios, constituyen un área importante para la investigación en marketing (Weitz \& Wensley, 2006). De acuerdo al estudio de Madrigal Moreno et al., (2018), las empresas del sector turístico de Morelia son conscientes de la importancia de estar presentes en las redes sociales virtuales ya que el $98 \%$ de ellas tiene presencia en al menos una.

Se le considera recurso intangible al capital relacional. Dicho activo se refiere a los intangibles generados por las relaciones que una empresa mantiene con los principales agentes económicos externos vinculados directamente con su actividad (Rodríguez Antón \& Rubio Andrada, 2006). El capital relacional se puede identificar también como los beneficios que le resultan al negocio de mantener buenas estrategias de relaciones con las partes interesadas del negocio. Dentro del capital relacional se encuentran autores que identifican el marketing relacional como una estrategia para la mejora, fortalecimiento y creación de relaciones que aportan valor a la empresa. Desde esta perspectiva, el marketing es el proceso de gestión de la organización en el mercado de las relaciones (Grönroos, 2004; Guzmán Duque, 2013).

El capital relacional se compone de tres elementos: relaciones con los clientes, relaciones con los proveedores y relaciones con los competidores. En el caso de los clientes y proveedores, los intercambios de información permiten a la empresa comprender mejor las necesidades de sus proveedores, pudiendo establecer así relaciones más estables y beneficiosas para ambas partes (Brooking, 1997). En la actualidad, sobre todo en la crisis que está viviendo el turismo, los competidores pueden convertirse en aliados estratégicos. Tal como lo señalaba Brooking (1997), el intercambio de cierta información, experiencias e inquietudes con otras empresas del mismo sector puede ayudar a mejorar la posición competitiva del grupo y permite afrontar problemas o proyectos que una sola empresa no podría abordar.

Por otra parte, diversos estudiosos de la mercadotecnia afirman que un negocio puede elaborar una estrategia exitosa de marketing si, en primer lugar, conoce a su cliente y elabora un perfil del mismo (Križman Pavlović \& Živolić, 2008). Es por ello que para la presente investigación se ha retomado el estudio del perfil del turista que elabora año tras año, desde el 2016, el Gobierno del Estado de Michoacán para la festividad del Día de Muertos. En el del año 2019, los resultados arrojaron que quienes visitaron la ciudad de Morelia fueron en su gran mayoría mexicanos, de los cuales, a su vez, la mayoría son del mismo Estado de Michoacán y cuentan con algún familiar en la ciudad (Gobierno de Michoacán, 2019). Esta información es de utilidad para los agentes involucrados en el sector del turismo de cara a elaborar sus estrategias de marketing.

\section{Los activos intangibles en la satisfacción del cliente en el servicio}

Desde el manejo de las quejas, hasta el servicio con una sonrisa, cuidar a los clientes es una ciencia en sí misma y no debe tomarse a la ligera. De otra forma puede tener un efecto dramático tanto en la moral del personal como en el resultado final. Abundan los estudios que muestran que aquellas empresas que obtienen buenos resultados en la experiencia del cliente tienen mayores ingresos y rendimientos de las inversiones (Naden, 2018). Los clientes no regresan a una empresa si tienen una mala experiencia. La información y servicio son elementos de la satisfacción del cliente, o en este caso del turista. Es por ello que tomaremos en cuenta la satisfacción del turista como factor del modelo de competitividad turística de (Crouch \& Brent Ritchie, 1999). 
El índice de satisfacción del turista y viajero en Michoacán es un estudio que lleva a cabo el Gobierno de Michoacán (2019). En el año 2018 levantó encuestas en la semana de las festividades de Día de Muertos, iniciativa que también tuvo lugar para esa semana durante el año 2019. En dicha encuesta se le pide al turista calificar 14 atributos del viaje en una escala del 1 al 10. Como se observa en la Tabla 1, la satisfacción del turista aumentó del 2018 al 2019.

Tabla 1. Índice de satisfacción del turista 2018-2019

Table 1. 2018-2019 tourist satisfaction index

\begin{tabular}{lcc}
\hline \multicolumn{1}{c}{ Atributos/Calificaciones } & Día de Muertos 2018 & Día de Muertos 2019 \\
\hline Satisfacción global del viaje & 8.85 & 8.97 \\
\hline Hospitalidad, trato, amigabilidad & 8.7 & 8.75 \\
\hline Gastronomía & 8.75 & 8.83 \\
\hline Diversión & 8.67 & 8.5 \\
\hline Limpieza urbana & 8.27 & 7.97 \\
\hline Calidad del alojamiento & 8.58 & 8.62 \\
\hline Conservación del entorno & 8.2 & 8.58 \\
\hline Información turística & 8.15 & 8.29 \\
\hline Percepción de seguridad pública & 8.45 & 8.15 \\
\hline Precios adecuados & 7.59 & 7.81 \\
\hline Transporte público & 7.75 & 7.75 \\
\hline Ruido ambiental & 7.35 & 7.65 \\
\hline Señalización & 7.85 & 8.05 \\
\hline Infraestructura (carreteras) & 7.51 & 7.88 \\
\hline
\end{tabular}

Fuente: elaboración propia a partir del estudio del Perfil del Turista (Gobierno de Michoacán, 2018; Gobierno de Michoacán, 2019).

Muchos de estos atributos responden a elementos intangibles (calidad, diversión, trato, satisfacción y percepción), elementos que se deben trabajar no solo desde las instituciones, sino también desde las empresas de servicios turísticos, cuestión que marca la orientación de esta investigación.

\section{METODOLOGÍA}

La presente investigación es cualitativa y los resultados derivan de una investigación documental. De acuerdo a Hernández Sampieri et al., (2010), la investigación cualitativa es un método no estructurado que busca proporcionar la idea y comprensión más profunda del problema. Este tipo de investigación pretende estudiar una parte de la realidad y no busca probar teorías o hipótesis, sino descubrir las cualidades del objeto de estudio. Dada la importancia del sector turístico para México, en términos económicos y sociales ya establecidos en el marco teórico de la investigación, surge la intención de estudiar el fenómeno de la competitividad turística, más aún del año 2019, sobre todo ahora si se tiene en cuenta que, por motivos de la pandemia de Covid-19, dicho sector se ha visto muy afectado. Para este estudio se analiza el caso de la ciudad de Morelia, ciudad que atrae turismo de tipo cultural. 
Este estudio cualitativo se fundamenta en un proceso exploratorio, en el cual se indaga a través de la revisión de la literatura encontrada sobre el tema de interés, la competitividad de un destino turístico. En la primera fase de la investigación se encuentra con el Reporte de Competitividad de Viajes y Turismo (Uppink Calderwood \& Soshkin, 2019). Es entonces que se puede identificar que los indicadores que el WEF utiliza para calcular la competitividad turística entre naciones son de naturaleza cuantitativa. El índice de competitividad es útil para calificar naciones, pero al momento de intentar utilizar ese mismo índice para conocer la competitividad de ciudades o comunidades pequeñas es difícil llegar a un cálculo correcto. Tal es el caso de una ciudad como Morelia. Es por ello que, en la búsqueda de un modelo que sea, por el contrario, de naturaleza cualitativa y de mejor comprensión en el estudio de ciudades, se encuentra el modelo de competitividad turística que proponen los investigadores (Crouch \& Brent Ritchie, 1999).

Teniendo en cuenta el contexto anterior, y de acuerdo a Kerlinger y Lee (2002), el problema de investigación establece la relación existente entre dos o más variables. En este caso, se busca fijar la relación entre la competitividad de las empresas del sector turístico con la de los activos intangibles vinculados con la organización, la mercadotecnia y la satisfacción del cliente, analizando su impacto en la sobrevivencia de las empresas del sector turístico de forma positiva. Los hallazgos sobre estos activos intangibles serán la base de conocimiento en grupos de interés como instituciones, empresas, investigadores, docentes y estudiantes.

Dado que los factores administrativos que contempla el modelo de Crouch y Brent Ritchie son de naturaleza intangible, para la investigación documental se han revisado libros y revistas científicas que aborden temas de intangibles en diversas bases de datos indexadas. Asimismo, para la conformación del marco teórico se han utilizado artículos encontrados en revistas científicas sobre la competitividad turística en el mundo. Finalmente, se elabora una propuesta de modelo de competitividad basada en activos intangibles.

Esta propuesta está fundamentada en la literatura encontrada en bases de datos tales como Emerald, Elsevier y Science Direct. La información obtenida busca aportar utilidad a los administradores de las empresas de la localidad, ya que podrán informarse de los activos intangibles que resulten más eficaces y que aporten competitividad a la organización, y así evitar el quiebre o desaparición de las mismas. Además, la propuesta de modelo de competitividad se podría replicar en otros sectores económicos de la ciudad de Morelia para apoyar las estrategias y políticas público-privadas de la localidad.

\section{RESULTADOS}

Como resultado de la búsqueda documental, se encontraron varios modelos de competitividad turística, tal como el del ranking del Foro Económico Mundial (Uppink Calderwood \& Soshkin, 2019) y el de (Crouch \& Brent Ritchie, 1999). En esta investigación se optó por enfocar el estudio en el modelo de Crouch y Brent Ritchie por sus características cualitativas, que, a diferencia del índice del Foro Económico Mundial no se basa en un modelo cuantitativo. Este modelo ha sido utilizado con éxito en estudios de competitividad turística en ciudades pequeñas de Italia (Cucculelli \& Goffi, 2016), Alemania (Hallmann et al., 2012) y, recientemente, retomado para estudiar la competitividad de ciudades anfitrionas en turismo de convenciones de Estados Unidos (Crouch et al., 2019). En este 
sentido, se parte del modelo de Crouch y Brent Ritchie (1999) como una referencia importante para el desarrollo de estrategias organizacionales en los negocios turísticos en la ciudad de Morelia, México.

Algunos académicos como Cronjé y du Plessis (2020) coinciden en que la creación del modelo de competitividad turística - presentado en 1999 y actualizado en 2003 por Brent Ritchie y Crouch, ha sido un aporte importante para el turismo, pues, comparado con otros modelos que sólo toman en cuenta el número de visitantes en un destino o su ocupación en el mercado, éste está integrado por múltiples factores que generan la competitividad del destino turístico, tales como la restricción, la atracción, el soporte y la administración (Ibáñez Pérez, 2011).

México obtuvo una calificación muy baja en seguridad en el reporte del (WEF, 2019). Al replicarse está situación en el resto del país, se considera que la inseguridad es el principal determinante restrictivo de la ciudad de Morelia para el turismo. No obstante, de acuerdo al estudio del perfil del turista (PERFITUR) realizado por Gobierno de Michoacán (2019), la percepción de seguridad del turista ha ido cambiando de manera positiva en los últimos años. Por otro lado, los factores básicos de atracción con los que cuenta la ciudad de Morelia en cuanto a cultura, son su centro histórico, patrimonio de la humanidad, y los eventos especiales que organiza la Secretaria de Turismo como son las festividades de Día de Muertos, el cual atrae turismo cultural nacional e internacional (Datatur, 2019).

Por otra parte, en relación con los factores de soporte del destino turístico de la ciudad de Morelia (integrados por la infraestructura aérea y terrestre, y la accesibilidad en cuestiones de TICS) existen múltiples deficiencias, tal como lo señala el reporte del (WEF, 2019). Le compete al Gobierno establecer mejorías en este sentido y así incidir favorablemente en el desarrollo social y económico de la comunidad. En los destinos donde el turismo es una actividad generadora de ingresos con efecto multiplicador, los integrantes de la comunidad se ven motivados a participar activamente en el proceso de desarrollo del mismo turismo (Cucculelli \& Goffi, 2016). El último de los factores del modelo es el que hace referencia a la administración de la empresa turística.

Después de analizar a la ciudad de Morelia bajo los factores del modelo de competitividad turística (Crouch \& Brent Ritchie, 1999), se reconoce que todos ellos son importantes para el destino turístico, y al mismo tiempo se identifica que los factores de restricción, atracción y soporte son de tipo cuantitativo, mientras que los de administración son de tipo cualitativo. Este trabajo se centra en el análisis de los factores de administración, considerando que la administración de una empresa de servicios turísticos está compuesta por activos intangibles. Este estudio pretende contribuir al modelo de competitividad turística y a los indicadores que forman parte de los factores de administración en dicho modelo para el turismo cultural de la ciudad de Morelia, México.

Diversos estudios en el área de la administración, tales como los de Aldehayyat y Twaissi (2011) y Bontis et al., (2015) plantean que un adecuado uso de los activos intangibles en los procesos de organización, mercadotecnia y satisfacción del cliente son aspectos de vital importancia en el desarrollo de la competitividad de dichas organizaciones. Por tanto, el estudio se adentra en el ámbito de estos activos intangibles como factores que deben considerarse para analizar la base competitiva de las organizaciones turísticas, teniendo en cuenta los modelos que se articulan alrededor del concepto «capital intelectual». 
Con base en los hallazgos de esta investigación documental, se identificó la relación existente entre los activos intangibles y la competitividad de la empresa, partiendo del modelo de competitividad de Crouch \& Brent Ritchie (1999) y de la teoría de recursos y capacidades (Barney, 1986; Grant, 1991; Kristandl \& Bontis, 2007). Tal como se observa en la Figura 2, se muestra la relación entre los activos intangibles de la organización, de la mercadotecnia y de la satisfacción del cliente con la competitividad turística de un destino, en este caso de la ciudad de Morelia.

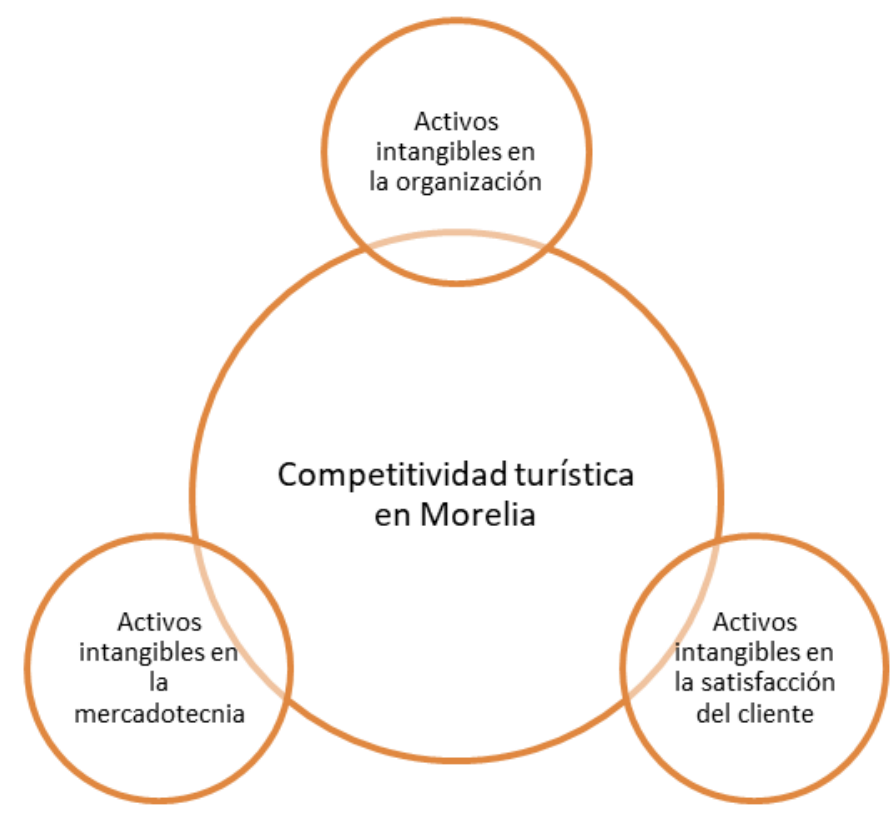

Figura 2. Activos intangibles que determinan la competitividad turística de la Ciudad de Morelia Figure 2. Intangible assets that determine the tourism competitiveness of the city of Morelia Fuente: elaboración propia con base en (Barney, 1986; G. Crouch \& Brent Ritchie, 1999; Grant, 1991; Kristandl \& Bontis, 2007).

En este sentido, tales intangibles se concentran en tres dimensiones: las personas, como centro de la capacidad intelectual (actitudes, aptitudes y experiencias) donde las organizaciones buscan contar con un grupo humano competente para potenciar el negocio; el conocimiento (por su naturaleza intangible), cuanto más se comparte más se enriquece (al contrario que los recursos tangibles que con su mayor uso más se deprecian); y una lectura estructural, corporativa, organizacional de los intangibles, para crear el ambiente, la agenda y los espacios y recursos que necesita el grupo humano para desarrollar sus intangibles (Bueno et al., 2011).

Estos intangibles se enmarcan en organizaciones con identificación y participación de sus grupos de interés, el establecimiento de alianzas, la participación en redes y plataformas nacionales e internacionales, $y$, finalmente, las estrategias de imagen (prestigio, notoriedad, reconocimiento) que pueden venir unidas a políticas de responsabilidad social empresarial con el territorio. En el caso de los intangibles, el perfil de los turistas y todos sus atributos relacionados generan un escenario de grupos de interés que es preciso identificar exhaustivamente, comprender sus motivaciones, necesidades e inquietudes para lo que es interesante vincularles, hacerles partícipes de procesos de 
mejora e innovación, seleccionando muestras específicas, utilizando canales formales e informales, virtuales y presenciales, además de crear dinámicas atractivas para fomentar dicha participación. La conformación del modelo de activos intangibles de la administración como factor de la competitividad turística se fundamenta en el Modelo Intellectus (Bueno et al., 2011), modelo de capital intelectual ampliamente aceptado en estudios de competitividad empresarial (GonzálezLoureiro \& Figueroa Dorrego, 2012; García-zambrano \& Rodríguez-Castellanos, 2017; Miles Touya, 2012; Rodríguez Antón \& Rubio Andrada, 2006). Dicho modelo considera como componentes del capital intelectual, el capital humano, el capital organizacional y el capital relacional. De este modelo se identifica que las características de intangibles de sus elementos coinciden con las características de intangibles que poseen los activos intangibles que conforman la administración de las empresas de servicios turísticos.

De esta manera es que se determina que el activo intangible de la organización se encuentra reflejado en el capital humano y organizacional; asimismo, el activo intangible de la mercadotecnia se ve reflejado en el capital relacional. Además, para identificar los elementos de la satisfacción del cliente como activo intangible, se han cruzado con los atributos del estudio PERFITUR teniendo en cuenta aquellos que tengan naturaleza intangible. Bajo la premisa de la teoría de recursos y capacidades de (Barney, 1986; Grant, 1991; Kristandl \& Bontis, 2007), se considera como intangible la satisfacción global del viaje, la hospitalidad, el trato y la amigabilidad, la gastronomía, la diversión y la información turística.

Tabla 2. Activos intangibles en la organización, mercadotecnia y satisfacción del cliente Table 2. Intangible assets in organization, marketing, and customer satisfaction

\begin{tabular}{|c|c|c|c|}
\hline Activos intangibles & Organización & Mercadotecnia & Satisfacción del cliente \\
\hline Conocimientos y capacidades & $x$ & & \\
\hline Valores y actitudes & $x$ & & \\
\hline Cultura organizacional & $x$ & & \\
\hline Estructura organizacional & $x$ & & \\
\hline Procesos organizacionales & $x$ & & \\
\hline Relaciones con los clientes y proveedores & & $x$ & \\
\hline Relaciones con los aliados & & $x$ & \\
\hline Satisfacción global del viaje & & & $x$ \\
\hline Hospitalidad, trato, amigabilidad & & & $x$ \\
\hline Gastronomía & & & $x$ \\
\hline Diversión & & & $X$ \\
\hline Información turística & & & $x$ \\
\hline
\end{tabular}

Fuente: elaboración propia a partir de (Bueno et al., 2011; González-Loureiro \& Figueroa Dorrego, 2012; García-zambrano \& Rodríguez-Castellanos, 2017; Gobierno de Michoacán, 2019; Miles Touya, 2012; Rodríguez Antón \& Rubio Andrada, 2006).

Los doce elementos que se muestran en la Tabla 2 conforman el modelo de competitividad turística de activos intangibles en la administración de empresas de servicios turísticos en el caso de estudio de la ciudad de Morelia, México. Dentro de la literatura se definen estos elementos. 
1. Conocimientos y capacidades: son propios del capital humano de la organización pero que contribuyen directamente en el buen desempeño de sus labores.

2. Valores y actitudes: definidos por la organización y seguidos por los miembros de la misma.

3. Cultura organizacional: ambiente en el que se desenvuelve el capital humano de la organización.

4. Estructura organizacional: es el diseño que tiene la organización para conformar su estructura.

5. Procesos organizacionales: que deben estar claramente definidos y conocidos por todos los miembros de la organización.

6. Relaciones con los clientes y proveedores: la manera en que la organización los trata; debe generar beneficios.

7. Relaciones con los aliados: contar con el apoyo de asociaciones e instituciones gubernamentales es importante para la organización.

8. Satisfacción global del viaje: directamente asociado al enfoque de satisfacción del cliente.

9. Hospitalidad, trato, amigabilidad: como atributo que se une a la satisfacción del cliente, además de a las competencias y políticas internas de trabajo.

10. Gastronomía: atributo que atrae al turista que busca conocer de sabores diferentes a los de su lugar de origen.

11. Diversión: como atributo que forma parte importante de la satisfacción del turista.

12. Información turística: considerado como atributo clave, siendo cada vez una variable más autónoma por parte del cliente dado su acceso a Internet.

Según los resultados de este estudio, el valor reside en incorporar una lectura micro de estos atributos, desde la dirección y gestión empresarial de las empresas del sector turístico, en este caso de Morelia, para que formen parte de sus estrategias. De esta forma, actúan como líneas o directrices para la mejora de la competitividad y no únicamente como atributos de una encuesta, teniendo en cuenta que son activos intangibles que es preciso incorporar en la agenda de trabajo de las organizaciones, siendo elementos normalmente «ocultos» o ignorados dado que se suelen plantear sistemas de dirección de corte económico, financiero y contable, muy alineados con los recursos tangibles.

\section{DISCUSIÓN}

La competitividad es un término que se ha estudiado desde que la Administración aún no nacía como ciencia, sino que estaba contenida en las Ciencias de la Economía. El origen de la noción de competitividad se remonta a Adam Smith, quien en "La naturaleza y causa de la riqueza de las Naciones», en 1776, enfatizó la importancia de producir a bajos costos, argumentando que la libertad de mercados determinaría de manera eficiente cómo la producción de un país podría satisfacer las necesidades de otros (González \& Mendieta, 2009). Casi dos siglos después, aparece «el modelo del diamante de la competitividad», establecido por Porter en el ámbito empresarial de la era moderna. Para (Porter \& Linde, 1995) la competitividad se determina por la productividad con la cual una nación, región o clúster utiliza sus recursos naturales, humanos y de capital. La productividad fija el estándar de vida de una nación o región (salarios, retornos al capital, retornos a las dotaciones de recursos naturales).

La ciencia está en constante avance, es por ello que los investigadores deben compartir toda innovación o mejora desde su área de estudio. Partiendo de teorías ya probadas y establecidas es 
que se pueden proponer innovaciones a las estrategias administrativas. El objetivo principal de esta investigación fue realizar una revisión exhaustiva de las investigaciones anteriores y actuales sobre la competitividad de los destinos turísticos para proporcionar una visión y claridad con relación a este trabajo. Tal como sostienen investigaciones recientes, entre ellos Cronjé y du Plessis (2020), los hallazgos clave de esta revisión revelan, entre otras cosas, que la mayoría de los estudios de casos de investigación se realizaron en Europa y, por lo tanto, enfatizan la necesidad de centrarse también en otros continentes. Es por ello la pertinencia de la presente investigación y la propuesta de modelo para medir la competitividad en una ciudad de América Latina.

El aporte de este trabajo, en el desarrollo de la línea de investigación que detona, lleva al terreno micro el planteamiento general que habitualmente se le aplica a los estudios sobre el sector turístico en México, ya sean iniciativas de Sectur, el Consejo de Promoción Turística de México, DataTur o la OCDE, en donde se analizan cuestiones como el perfil del viajero, los países de procedencia, la tipología de turismo, la duración de las estancias, los ingresos derivados, e incluso, un enfoque descriptivo sobre el número de hoteles, tipología de establecimientos, inversiones, balanza comercial, etcétera. Todo ello incide en las políticas públicas, en los denominados policy makers, pero falta una mirada mucho más cercana a la empresa, a la labor directiva y gestora.

La mejora de la propuesta de valor de las organizaciones no pasa por contar con acceso a información sobre macromagnitudes, ni tampoco por identificar necesidades desatendidas o insatisfechas en los clientes actuales o potenciales, sino por crear organizaciones que son capaces de progresar (responder y anticipar), basando dicho progreso en el aprovechamiento del conocimiento disponible interna y externamente bajo condiciones organizativas de motivación, cohesión y aprendizaje en un entorno abierto a conexiones enriquecedoras con agentes externos. Este es el auténtico perfil diferencial de este estudio frente a las perspectivas habituales de análisis del sector turístico en México.

El fortalecimiento real de este sector en México vendrá procedente del punto de encuentro entre el enfoque Top-Down de las políticas públicas, y el manejo de la dimensión macro y el enfoque Bottomup que proviene de las estrategias empresariales, para así hacer confluir dos ejes de cara a potenciar la competitividad del sector turístico, creando directrices, condiciones, competencias y propuestas de valor en sintonía.

El caso de Morelia es muy representativo como destino turístico cultural y de costumbres, pues no solo va más allá del llamado turismo "sol y playa», sino que atesora un vector de desarrollo de alta dimensión para el país, no sólo por ser un mercado menos conocido con más potencial de descubrimiento, sino por su mencionado carácter de tracción económica directa, y sobre todo indirecta, de cara a generar progreso y bienestar en la sociedad mexicana, en línea con los objetivos de desarrollo sostenible (ODS).

Por tanto, la línea de investigación comienza aquí con un marco de referencia de factores de competitividad y su relación con los intangibles que lleva a una implicación práctica derivada de la creación de un modelo de competitividad aplicable a las empresas del sector turístico - contando con el caso de Morelia como acción piloto - para lo que se requiere establecer un modelo de análisis que avance el marco de referencia de PERFITUR con el cual generar un cuestionario y un trabajo de campo exhaustivo con agentes del sector turístico en Morelia. 


\section{CONCLUSIONES}

Como resultado de la investigación documental, se concluye que los activos intangibles son factores importantes en la construcción de la competitividad de los destinos turísticos y, más aún, pueden garantizar el éxito en los mercados competitivos de la actualidad. Los factores de competitividad identificados asociados a la administración (organización, mercadotecnia y satisfacción del cliente) y sus intangibles vinculados, pueden generar unas estrategias empresariales específicas que traduzcan los estudios, sus resultados y recomendaciones macro en directrices útiles para las empresas del sector turístico.

El de los activos intangibles es un enfoque poco utilizado en las estrategias administrativas, por tal motivo, estudios como el que aquí se presenta, buscan aportar conocimiento sobre el tema. Con base a los resultados del estudio de PERFITUR respecto a la satisfacción del turista en Morelia y sus alrededores, se puede concluir que los turistas son cada vez más exigentes con el destino al que viajan, es por ello que los prestadores de servicios turísticos deben poner mayor énfasis en la atención al turista para lograr la satisfacción del mismo y conseguir su fidelidad.

De la lista de 14 atributos que califican los turistas para expresar su satisfacción del viaje, existen algunos que les compete desarrollar totalmente a los servidores de servicios turísticos, como son la hospitalidad, el trato, la amigabilidad, la gastronomía y la calidad del alojamiento. De ahí la importancia de que el sector turismo se informe sobre modelos y estrategias de servicio al cliente para contribuir a la competitividad de su empresa. Por otro lado, la diversión y limpieza urbana, conservación del entorno, información turística, percepción de seguridad pública, señalización e infraestructura, transporte público y ruido ambiental son factores que le competen desarrollar al Gobierno municipal, estatal y federal.

Es de vital importancia que este, y todos los estudios que se hagan sobre el turismo, sean de dominio público, que exista un vínculo entre la academia, los prestadores de servicios turísticos y la Secretaria de Turismo. El sector turismo aporta 4 millones de empleos en México de manera directa y otros 6 millones de manera indirecta, de ahí la importancia de seguir investigando el área de turismo. En momentos donde la economía del país está en bajo crecimiento, sobre todo en el sector turismo debido a la pandemia, todo estudio que proponga conocimiento nuevo para obtener alguna ventaja competitiva será vital.

En el siglo XXI, dos inmensos motores de cambio para la industria del turismo son el cambio climático y las emergencias sanitarias mundiales. Un ejemplo de tal emergencia es el COVID-19 que circula actualmente y que ha ocasionado el cierre de actividades turísticas (Jamal \& Budke, 2020). Es muy importante que dentro de cualquier estrategia administrativa se tome en cuenta esta o alguna futura pandemia. El sector turismo está en un proceso de aprendizaje al verse obligado a reconfigurar sus protocolos de seguridad e higiene, y la academia debe ver esta situación como un punto de oportunidad para futuras investigaciones del tema.

\section{CONFLICTOS DE INTERÉS}

Los autores declaran que no presentan conflictos de interés financiero, profesional o personal que pueda influir de forma inapropiada en los resultados obtenidos o las interpretaciones propuestas. 


\section{CONTRIBUCIÓN DE AUTORES}

Para el desarrollo de este proyecto todos los autores han realizado una contribución significativa especificada a continuación:

Yuselim Angélica Villa-Hernández: desarrolló la investigación documental, la revisión de la literatura, además de fundamentar de manera conceptual el modelo propuesto en el artículo y finalmente, atender la revisión del editor.

Carlos Merino-Moreno: contribuyó en la investigación aportando literatura actual y con el diseño del modelo propuesto en el artículo, además de atender la estandarización de la información presentada.

Virginia Hernández-Silva: aportó valiosos datos secundarios obtenidos de organizaciones gubernamentales y en el desarrollo del apartado metodológico.

\section{REFERENCIAS}

Aldehayyat, J.; Twaissi, N. (2011). Strategic Planning and Corporate Performance Relationship in Small Business Firms: Evidence from a Middle East Country Context. International Journal of Business and Management, v. 6, n. 8, 255-263. https://doi.org/10.5539/ijbm.v6n8p255

Báez-Montenegro, A.; Devesa-Fernández, M. (2017). Motivation, satisfaction and loyalty in the case of a film festival: differences between local and non-local participants. Journal of Cultural Economics, v. 41, n. 2, 173-195. https://doi.org/10.1007/s10824-017-9292-2

Barber, J. P.; Darder, F. L. (2004). Dirección de Empresas Internacionales. Pearson Educación.

Barney, J. B. (1986). Organizational culture: Can it be a source of sustained competitive advantage? Academy of Management Review, v. 11, n. 3, 656-665. https://doi.org/10.5465/amr.1986.4306261

Bobirca, A.; Cristureanu, C. (2015). Analyzing Romania's competitiviness as a tourism destination. En Chen, J.S. (Ed.), Advances in Hospitality and Leisure (v. 4 pp. 75-99). Emerald Group Publishing Limited. https://doi.org/10.1016/S1745-3542(08)00004-0

Bontis, N. (2001). Managing organizational knowledge by diagnosing intellectual capital; framing and advancing the state of the field. In Knowledge Management and Business Model Innovation, 433-462. https://doi.org/10.4018/978-1-878289-98-8.ch016

Bontis, N.; Janošević, S.; Dženopoljac, V. (2015). Intellectual capital in serbia's hotel industry. International Journal of Contemporary Hospitality Management, v. 27, n. 6, 1365-1384. https://doi.org/10.1108/IJCHM-12-2013-0541

Brent Ritchie, J. R.; Crouch, G. I. (2003). The Competitive Destination, a Sustainable Tourism Perspective. CABI Publishing. 
Brooking, A. (1997). El capital intelectual. El principal activo de las empresas del tercer milenio. (P. I. S. A. (ed.).

Bueno Campos, E. (1998). El Capital Intangible como Clave Estratégica en la Competencia Actual. Boletín de Estudios Económicos, v. 53, n. 164, 207-229. URL

Bueno, E.; del Real, H.; Fernández, P.; Longo, M.; Merino, C.; Murcia, C.; Salmador, M. P. (2011). Modelo Intellectus: Medición y gestión del capital intelectual. Universidad Autónoma de Madrid.

Bueno, E.; Salmador, M. P.; Merino, C. (2008). Génesis, concepto y desarrollo del capital intelectual en la economía del conocimiento: Una reflexión sobre el Modelo Intellectus y sus aplicaciones. Estudios de Economía Aplicada, v. 26, n. 2, 43-63. URL

Chen, J.; Zhu, Z.; Yuan Xie, H. (2004). Measuring intellectual capital: a new model and empirical study. Journal of Intellectual Capital, v. 5, n. 1, 195-212. https://doi.org/10.1108/14691930410513003

Chu, C.; Uebegang, K. (2002). Saving Hong Kong's Cultural Heritage. Civic Exchange. URL

Consejo Nacional Empresarial Turístico; Centro de Investigación y Competitividad Turística Anáhuac. (2020). Estimación de las afectaciones al turismo mexicano en 2020 como consecuencia de la pandemia de Covid-19. URL

Coordinación de Asesores del Secretario de Turismo. (2018). Nuestro Turismo., el gran motor de la economía nacional. Secretaría de turismo. $\underline{\mathrm{URL}}$

Corte-Cruz, P. S. (2019). La demanda agregada de la cultura en México: propensión al consumo y tasa de interés para empresas culturales. Revista CEA, v. 5, n. 10, 151-165. https://doi.org/10.22430/24223182.1331

Cronjé, D. F.; du Plessis, E. (2020). A review on tourism destination competitiveness. Journal of Hospitality and Tourism Management, v. 45, 256-265. https://doi.org/10.1016/j.jhtm.2020.06.012

Crouch, G. I.; Brent Ritchie, J. R. (1999). Tourism, Competitiveness, and Societal Prosperity. Journal of Business Research, v. 44, n. 3, 137-152. https://doi.org/10.1016/S0148-2963(97)00196-3

Crouch, G. I.; Del Chiappa, G.; Perdue, R. R. (2019). International convention tourism: A choice modelling experiment of host city competition. Tourism Management, v. 71, 530-542. https://doi.org/10.1016/j.tourman.2018.10.002

Cucculelli, M.; Goffi, G. (2016). Does sustainability enhance tourism destination competitiveness? Evidence from Italian Destinations of Excellence. Journal of Cleaner Production, v. 111, 370-382. https://doi.org/10.1016/i.jclepro.2014.12.069

DATATUR. (2019). Ciudades Mexicanas Patrimonio Mundial. $\underline{\text { URL }}$ 
Djukic, A.; Vukmirovic, M. (2012). Creative cultural tourism as a function of competitiveness of cities. Technics Technologies Education Management, v. 7, n. 1, 404-410. URL

El Universal. (2020, 10 de octubre). Destinos turísticos se unen para armonizar protocolos contra Covid-19. URL

Fitz Patrick, M.; Davey, J.; Muller, L.; Davey, H. (2013). Value-creating assets in tourism management: Applying marketing's service-dominant logic in the hotel industry. Tourism Management, v. 36, 86-98. https://doi.org/10.1016/i.tourman.2012.11.009

García-Gallo, M. D.; Jiménez-Naharro, F.; Torres-García, M.; Giesecke, S. L.; Guadix-Martín, J. (2020). Incorporation of the Intangibles into the Spanish Start-Ups by Activity Sector and Region. Improving Their Economic Sustainability. Sustainability, v. 12, n. 10, 4268. https://doi.org/10.3390/su12104268

García-Zambrano, L.; Rodríguez-Castellanos, A. (2017). Capital intelectual y resultados empresariales: vinculación de competencias empresariales en el caso español. Memorias, v. 15, n. 27, 105-118. $\underline{U R L}$

Gobierno de Michoacán. (2018). Perfil y grado de satisfacción de los turistas que visitan Michoacán. Noche de Muertos 2018. URL

Gobierno de Michoacán. (2019). Resultados 2019. Noche de Muertos. URL

Gómez-Bayona, L.; Londoño-Montoya, E.; Mora-González, B. (2020). Modelos de capital intelectual a nivel empresarial y su aporte en la creación de valor. Revista CEA, v. 6, 11, 165-184. https://doi.org/10.22430/24223182.1434

Gómez Vega, M.; Herrero Prieto, L. C. (2017). Determinantes de la eficiencia en la captación de turismo cultural nacional y extranjero en España: Un análisis regional. Estudios de Economía Aplicada, v. 35, n. 3, 849-872. URL

González, R. C.; Mendieta, M. D. (2009). Reflexiones sobre la conceptualización de la competitividad de destinos turísticos. Cuadernos de Turismo, n. 23, 111-128. URL

González-Loureiro, M.; Figueroa Dorrego, P. (2012). Intellectual capital and system of innovation: What really matters at innovative SMEs. Intangible Capital, v. 8, n. 2, 239-274. URL

Grant, R. M. (1991). The resource-based theory of competitive advantage: Implications for strategy formulation. California Management Review, v. 33, n. 3, 114-135. https://doi.org/10.2307/41166664

Grönroos, C. (2004). The relationship marketing process: communication, interaction, dialogue, value. Journal of Business \& Industrial Marketing, v. 19, n. 2, 99-113. https://doi.org/10.1108/08858620410523981 
Guzmán Duque, A. P. (2013). Factores críticos de éxito en el uso de las redes sociales en el ámbito universitario: aplicación a Twitter [Tesis de doctorado, Universidad Politécnica de Valencia]. https://doi.org/10.4995/Thesis/10251/28212

Hallmann, K.; Müller, S.; Feiler, S.; Breuer, C.; Roth, R. (2012). Suppliers' perception of destination competitiveness in a winter sport resort. Tourism Review, v. 67, n. 2, 13-21. https://doi.org/10.1108/16605371211236105

Hamel, G.; Prahalad, C. K. (1997). The core competence of the corporation. In Hahn, D.; Taylor B. (Eds) Strategische Unternehmungsplanung / Strategische Unternehmungsführung. Physica. https://doi.org/10.1007/978-3-662-41482-8 46

Hassan, S. S. (2000). Determinants of Market Competitiveness in a environmentally sustainable tourism industry. Journal of Travel Research, v. 38, n. 3, 239-245. https://doi.org/10.1177/004728750003800305

Hernández Sampieri, R.; Fernández Collado, C.; Baptista Lucio, P. (2010). Metodología de la Investigación. McGraw Hill.

Hernández, S. (2011). Introducción a la Administración (5ed.). McGraw Hill.

Hoyos, R. (2008). Marketing: más de 100 años de historia. Génesis y evolución de un concepto. Asomercadeo, Lecturas Seleccionadas de Mercadeo.

Ibáñez Pérez, R. M. (2011). Diagnóstico de la calidad y competitividad del sector turístico en México. Cuadernos de Turismo, n. 28, 121-143. URL

Instituto Nacional de Estadística y Geografía. (2016). Datos. Esperanza de vida de los negocios por entidad federativa. $\underline{\mathrm{URL}}$

Jamal, T.; Budke, C. (2020). Tourism in a world with pandemics: local-global responsibility and action. Journal of Tourism Futures, v. 6, n. 2, 181-188. https://doi.org/10.1108/JTF-02-2020-0014

Kerlinger, F. N.; Lee, H. B. (2002). Investigación del comportamiento. McGraw-Hill.

Kristandl, G.; Bontis, N. (2007). Constructing a definition for intangibles using the resource based view of the firm. Management Decision, v. 45, n. 9, 1510-1524. https://doi.org/10.1108/00251740710828744

Križman Pavlović, D.; Živolić, S. (2008). Tourism destination marketing management: status and prospects in Croatia. Economic research-Ekonomska istraživanja, v. 21, n. 2, 99-113. URL

Larina, Y. S.; Romanenko, O. O. (2015). A New Role of Marketing and Communication Technologies in Business and Society: local and global aspects. Khusar Group. https://doi.org/10.13140/RG.2.1.2858.1607 
Madrigal Moreno, S.; Alfaro Calderón, G. G.; Madrigal Moreno, F. (2018). Apropiación de las redes sociales en el sector turístico en Morelia, México. Revista Espacios, v. 39, n. 19. URL

Migueles, R. (2020, 10 de octubre). Los ingresos por turismo internacional crecen $3.8 \%$ URL

Miles Touya, J. G. (2012). Análisis del capital intelectual de las pequeñas y medianas empresas uruguayas y su impacto en los resultados. Un estudio en las empresas desarrolladoras de software. Un enfoque en el capital directivo emprendedor [Tesis doctoral, Universidad de Deusto]. $\underline{U R L}$

Miles, J.; Quintillán, I. (2005). Auto-evaluación del capital intelectual en la pequeña y mediana empresa. Revista Electrónica FCE, 2-28.

Naden, C. (2018). Improving customer satisfaction with updated ISO series of standards. URL

Ochoa Hernández, M. L. L.; Prieto Moreno, M. B.; Santidrián Arroyo, A. (2012). Una revisión de las principales teorías aplicables al capital intelectual. Revista Nacional de Administración, v. 3, n. 2, 35-48. https://doi.org/10.22458/rna.v3i2.486

Organización Mundial del Turismo. (2020, 27 de octubre). El turismo internacional cae un 70\% mientras las restricciones de viaje afectan a todas las regiones. $\underline{U R L}$

Porter, M. E.; Linde, C. V. D. (1995). Toward a New Conception of the Environment-Competitiveness Relationship. Journal of Economic Perspectives, v. 9, n. 4, 97-118. https://doi.org/10.1257/jep.9.4.97

Rodríguez Antón, J. M.; Rubio Andrada, L. (2006). Propuesta de creación de un modelo de capital intelectual hotelero latinoamericano. Estudios Turísticos, n. 167, 55-90. URL

Shaw, E. H.; Brian Jones, D. G. (2005). A history of schools of marketing thought. Marketing Theory, v. 5, n. 3, 239-281. https://doi.org/10.1177/1470593105054898

Teixeira, S. J.; Ferreira, J. J. M.; Almeida, A.; Parra-Lopez, E. (2019). Tourist events and satisfaction: a product of regional tourism competitiveness. Tourism Review, v. 74, n. 4, 943-977. https://doi.org/10.1108/TR-12-2018-0181

Unidad de Asuntos y Cooperación Internacionales. (2020). Resultados de la Actividad Turística Agosto 2020. Secretaría de Turismo. URL

Uppink Calderwood, L.; Soshkin, M. (2019). The travel \& tourism competitiveness Report 2019. Travel and tourism at a Tipping Point. World Economic Forum.

Weitz, B. A.; Wensley, R. (2006). Handbook of marketing. SAGE Publications.

World Economic Forum. (2019). Reports. Travel \& Tourism Competitiveness Index. URL 
Competitividad en el turismo cultural: estudio de caso

Zaragoza-Sáez, P. C.; Claver-Cortés, E.; Marco-Lajara, B.; Úbeda-García, M. (2020). Corporate social responsibility and strategic knowledge management as mediators between sustainable intangible capital and hotel performance. Journal of Sustainable Tourism, 1-23.

https://doi.org/10.1080/09669582.2020.1811289 